\title{
Impacto de la combustión del transporte terrestre en la calidad del aire y la sa- lud pública en áreas urbanas. Una revisión
}

\author{
Impact of Land Transport Combustion on air quality \\ and public health in urban areas. A Review
}

Angie Catherine Patiño-Sánchez*, @ https://orcid.org/0000-0001-9213-3975

y Omar Francisco Patiño-Silva**; ${ }^{*}$ (ittps://orcid.org/0000-0002-2318-2498

Citar este artículo como: Patiño-Sánchez, A. C. y Patiño-Silva, O. F. (2021). Impacto de la combustión del transporte terrestre en la calidad del aire y la salud pública en áreas urbanas. Una revisión. Revista Nodo, 15(30), pp. 61-73.

\section{Resumen}

El transporte terrestre, en la actualidad, es una necesidad; su funcionamiento es generado principalmente por el uso de combustibles fósiles que, por medio de una combustión incompleta en los vehículos automotores, producen la energía necesaria para su movimiento así como la emisión de monóxido de carbono, dióxido de carbono, óxidos de nitrógeno, óxidos de azufre y compuestos orgánicos volátiles que, al entrar en contacto con el ser humano, son consideradas las principales causas de las enfermedades respiratorias, cancerígenas y sanguíneas. En este sentido, en el presente artículo se realiza un compilado de las investigaciones efectuadas a nivel global, que permitan determinar la relación entre la combustión asociada con el funcionamiento del transporte terrestre y los niveles de contaminación que afectan la calidad del aire y la salud de las personas. De acuerdo con las investigaciones a nivel global, se encuentra que el material particulado (PST, $\mathrm{PM}_{10}$ y $\mathrm{PM}_{2,5}$ ) es el principal contaminante relacionado con la influencia del transporte terrestre en la salud pública.

Palabras clave: Contaminación ambiental, Combustible, Transporte urbano, Salud, Zona urbana.

\section{Abstract}

Land transportation is a necessity today, whose operation is generated mainly by the use of fossil fuels, which through incomplete combustion in motor vehicles generate the energy necessary for their movement, as well as the emission of carbon monoxide, carbon dioxide, nitrogen oxides, sulfur oxides and volatile organic compounds, which in contact with humans are considered the main causes of respiratory, carcinogenic and blood

Fecha de recepción: 6 de junio de 2020 • Fecha de revisión: 4 de octubre de 2020

Fecha de aceptación: 15 de diciembre de 2020 • Fecha de publicación: 9 de abril de 2021

\footnotetext{
* Magíster en Desarrollo Sustentable y Gestión Ambiental, Facultad de Medio Ambiente y Recursos Naturales, Universidad Distrital Francisco José de Caldas. Correo electrónico: acpatinos@correo.udistrital.edu.co; angiepatinosanchez@gmail.com

** Magíster en Gestión Ambiental, Docente de la Universidad Distrital Francisco José de Caldas, Facultad de Medio Ambiente y Recursos Naturales. Correo electrónico: ofpatinos@udistrital.edu.co
} 
diseases. In this sense, in this article a compilation of the investigations carried out at a global level is made, which will determine the relationship between the combustion associated with the operation of land transport and the levels of pollution that check air quality and public health. According to global research, it is found that particulate matter (PST, $\mathrm{PM}_{10}$ and $\mathrm{PM}_{2.5}$ ) is the main pollutant that is related to the influence of land transport on public health.

Keywords: Environmental pollution, Fuel, Urban transport, Health, Urban Zone.

\section{Introducción}

El funcionamiento del transporte terrestre convencional se caracteriza por el uso de combustibles fósiles, específicamente derivados del petróleo, a partir de los cuales se generan emisiones de Monóxido de carbono (CO), Dióxido de Carbono $\left(\mathrm{CO}_{2}\right)$, Óxidos de nitrógeno $\left(\mathrm{NO}_{\mathrm{x}}\right)$, Óxidos de azufre $\left(\mathrm{SO}_{\mathrm{X}}\right)$ y Compuestos Orgánicos Volátiles (VOC's, por sus siglas en inglés) que, al entrar en contacto con el ser humano, son consideradas las principales causas de enfermedades respiratorias, cancerígenas y sanguíneas (Gómez y Vigano, 2007). La afectación por parte de estas partículas depende de su tamaño, siendo las de menor diámetro, las que ingresan con mayor facilidad por las vías respiratorias (Arciniégas, 2012).

A nivel mundial, el tráfico vehicular es el responsable de una cuarta parte de los niveles de contaminación en el aire urbano en general, no obstante, en el sur y sudeste de Asia, América del Sur y Suroeste de Europa, la proporción de la concentración de contaminantes atribuida al tráfico vehicular, es del 30\% al 37\% (Karagulian et al., 2015, citado por Martins y Carrilho, 2018).

Con el fin de identificar el impacto de la combustión del transporte terrestre en la calidad del aire y la salud pública, se utiliza una metodología de búsqueda sistemática, tomando como referentes las metodologías implementadas en las investi- gaciones de Zambrano y Rojas (2008) donde se identifica el problema de la contaminación del aire en ciudades de México con respecto a la planeación urbana; de la misma forma Cursach, Rau, Tobar y Ojeda, J. (2012) por medio de una revisión bibliográfica determinó el estado actualizado del conocimiento sobre ecología urbana en las grandes ciudades del sur de Chile. Adicionalmente, en ciudades de Brasil la búsqueda sistemática de información ha sido utilizada en investigaciones asociadas a la caracterización de conurbaciones, como en el caso de Mendoza y Lombardo (2009). En otro contexto, la revisión sistemática de información ha sido útil en el estudio de variables asociadas a la calidad de recursos tales como el agua como se evidencia en la investigación de Jurado y Mercado (2017).

\section{Materiales y métodos}

Con el fin de realizar la revisión bibliográfica se utilizó una metodología de búsqueda sistemática de los estudios realizados a nivel mundial, acerca de la calidad del aire en las áreas urbanas. Las bases de datos utilizadas para identificar los documentos pertinentes, tales como artículos y libros, fueron Google Académico, Science Direct y Scopus. Adicionalmente, se utilizaron las siguientes palabras clave: "calidad de aire", "contaminantes", "combustibles fósiles" y "transporte terrestre". Los documentos detectados se limitaron temporalmente del año 2007 al 2017.

Por medio de esta búsqueda sistemática se identificó un total de 1610 documentos, de los cuales el 28\% corresponde a documentos en Google Académico; 33,5\% en Science Direct y 38,5\% en Scopus, para lo que se aplicaron filtros de exclusión en temas relacionados con artículos técnicos de salud y medicina, así como en temáticas en las que la discusión central se asocia con el etanol y la energía eléctrica para las actividades de transporte terrestre.

Posteriormente se seleccionaron los documentos por medio de los cuales se estableció el grado de 
contaminación en el aire, que se genera a partir de los procesos de combustión en vehículos de transporte terrestre convencional a nivel mundial, teniendo en cuenta la densidad poblacional de la zona de evaluación. De los documentos encontrados se excluyen los que presentan como base una temática social y los que describen la situación evaluada solamente en términos cualitativos, mientras que prevalecieron los autores con un mayor número de citas, por lo que la cantidad de información se reduce aproximadamente en $90 \%$.

Para realizar dicha selección, los documentos se calificaron de acuerdo a su pertinencia con respecto a las siguientes temáticas relacionadas con la calidad del aire: i) combustibles fósiles, ii) calidad de los combustibles, iii) contaminantes, iv) áreas urbanas y densidad poblacional y v) transporte terrestre; ello se realizó a través de la revisión de los artículos respectivos.

Una vez se seleccionaron los documentos se establecieron las categorías que facilitaron el análisis de la discusión: i) metodologías y herramientas, ii) tipo de contaminantes identificados iii) influencia de dichos contaminantes en la calidad del aire general de la zona de estudio (Tabla 1).
De acuerdo con esta selección se desarrolló una última fase de análisis con la bibliografía detectada, aplicando parámetros estadísticos; así mismo, con las concentraciones de los contaminantes identificados, se realizó una comparación con la normatividad ambiental que regula las megaciudades del mundo.

\section{Resultados y discusión}

El transporte terrestre convencional se caracteriza por el funcionamiento del motor con gasolina (octano) o diesel (dodecano), que corresponde a una mezcla de hidrocarburos derivados del petróleo; esta clase de combustible se oxida en presencia de aire, liberando una gran cantidad de energía por medio de una reacción de combustión (Cengel, 2006). Adicionalmente este tipo de combustible se compone de aditivos y catalizadores, moléculas de azufre, compuestos aromáticos y parafinas (Arango, 2009).

Como producto de la reacción de combustión incompleta se obtiene la liberación de energía, así como las emisiones de hidrocarburos (HC), Óxidos de Nitrógeno $\left(\mathrm{NO}_{\mathrm{x}}\right)$, Óxidos de Azufre

Tabla 1. Categorías de análisis para la información bibliográfica seleccionada.

\begin{tabular}{|c|c|c|}
\hline Categoría & Subcategoría & Documentos (\%) \\
\hline \multirow{2}{*}{ Metodologías y herramientas } & (i) Metodologías cuantitativas & 90 \\
\hline & (iii) Herramientas de medición & 90 \\
\hline \multirow{7}{*}{ Contaminantes } & (i) $\mathrm{PM}_{10}$ & 85 \\
\hline & (ii) $\mathrm{PM}_{2,5}$ & 85 \\
\hline & (iii) PST & 85 \\
\hline & (iv) Óxidos de Azufre & 15 \\
\hline & (v) Partículas Ultrafinas & 16 \\
\hline & (vi) Monóxido de Carbono & 14 \\
\hline & (vii) Óxidos de Nitrógeno & 15 \\
\hline \multirow{3}{*}{ Influencia de Contaminantes } & (i) Toxicidad & 90 \\
\hline & (ii) Paisaje & 10 \\
\hline & (iii) Normatividad Megaciudades & 45 \\
\hline
\end{tabular}

Fuente: elaboración propia. 
( $\left.\mathrm{SO}_{\mathrm{x}}\right)$, Monóxido de Carbono (CO) y Dióxido de Carbono $\left(\mathrm{CO}_{2}\right)$, compuestos que en su mayoría son tóxicos y representan un serio impacto en la salud pública, por la concentración de los contaminantes en la atmósfera (Llanes, Rocha, Peralta y Leguísamo, 2018).

De las emisiones generadas, el mayor impacto en la salud humana se define por el tamaño de las partículas; las menores a 10 micrómetros $\left(\mathrm{PM}_{10}\right)$ están relacionadas con la combustión de diesel, mientras que las inferiores a 2,5 micrómetros $\left(\mathrm{PM}_{2.5}\right)$ consiste en compuestos asociado, en general, a partículas ácidas por la combustión de combustibles fósiles. Los efectos en la salud están relacionados con problemas en las vías respiratorias, reacciones alérgicas, cáncer de pulmón, así como riesgos cardiovasculares (Gaviria, Benavides y Tangarife, 2011).

Las mediciones de material particulado se realizan por medio de un equipo muestreador que recolecta $\mathrm{PM}_{10}$ y $\mathrm{PM}_{2.5}$ a través de un filtro, determinando el peso de las partículas por medio de técnicas gravimétricas; mientras que la determinación de $\mathrm{SO}_{x}$ y NOx se realiza por una solución adsorbente, posteriormente cuantificado en el laboratorio (Hao et al., 2018), siguiendo las metodologías establecidas por la US-EPA, Título 40, parte 50.

La normatividad colombiana establece los valores máximos permisibles para cada contaminante del aire en la Resolución 2254 de 2017, expedida por el Ministerio de Ambiente y Desarrollo Sostenible; mientras que, en Estados Unidos, la normativa es establecida por la US-EPA, Título 40, parte 50. La normativa europea regula la calidad del aire por medio de la Directiva 2008/50/CE, Directiva 2004/107/CE y Directiva 2015/1480/CE.

\section{Emisiones, densidad poblacional y salud}

La investigación bibliográfica arrojó resultados de emisiones de los procesos de combustión en la actividad de transporte terrestre por parte de moléculas de Partículas Suspendidas Totales (PST), $\mathrm{PM}_{10}, \mathrm{PM}_{2.5}$, Partículas Ultrafinas (UFP), $\mathrm{CO}, \mathrm{NO}_{2}$, $\mathrm{NO}_{\mathrm{x}}$, NO, Carbono Negro, Carbono Elemental y $\mathrm{SO}_{2}$, en ciudades como Beijing (China), Bogotá (Colombia), Río de Janeiro (Brasil), Brisbane (Australia), Buenos Aires (Argentina), El Cairo (Egipto), Ciudad Heredia (Costa Rica), Guadalajara (México), Hangzhou (China), Madrid (España), Mashhad (Irán), Monasterevin (Irlanda), Nueva Delhi (India), Singapur (República de Singapur), Quito (Ecuador), Taichung (Taiwan) y Yokohama (Japón).

\section{Contaminación por material particulado $\left(\mathbf{P M}_{10}, \mathbf{P M}_{2,5}\right.$ y UFP)}

El material particulado corresponde a las partículas líquidas que tienen la capacidad de infiltrarse en las vías respiratorias; es producido en las áreas urbanas por varios factores antropogénicos y naturales, entre los cuales se encuentra la actividad de transporte terrestre. En el caso de Bogotá, en particular, la problemática del transporte se asocia a factores como el tipo de combustible, la edad y la tecnología de los vehículos, las conductas de conducción, el número de vehículos en servicio y la densidad de la población (Villalobos, Sánchez y Rodríguez, 2016).

De manera global, a partir de la investigación bibliográfica se destaca el caso de China en donde según Le, Yun, Yi-Ping, Mei-Bian y Dean (2012), aumenta la preocupación con respecto a la presencia de material particulado en el aire, ya que la medición del Îndice de Calidad (API), que involucra el $\mathrm{PM}_{10}$ (Partículas menores a 10 $\mu \mathrm{m})$, incumple con la normatividad establecida por el Ministerio de Medio Ambiente de China); sin embargo, no se regulan las emisiones de la presencia de $\mathrm{PM}_{2.5}$ (Partículas menores a 2,5 $\mu \mathrm{m}$ ), material que afecta aún más la salud debido al menor tamaño diámetro. De la misma forma en el artículo de Le et al. (2012), se expone la importancia de la medición de las partículas ultrafinas (UFP) que corresponden a partículas de diámetro menor a $0,1 \mu \mathrm{m}$, las cuales han sido 
poco estudiadas y no reguladas; sin embargo, a partir de este estudio, su concentración presenta una influencia significativa por parte de las condiciones meteorológicas.

De la misma forma, O'Donoghue, Broderick y Delaney (2007) resaltan la importancia del material particulado e identifican una gran parte de su origen en los automóviles, lo que genera enfermedades respiratorias como el asma y patologías cardiovasculares.

En Ciudad de México, algunos estudios realizados también se han interesado por la calidad del aire, en especial por las Partículas Ultrafinas (UFP) ya que, debido a su tamaño, fácilmente pueden entrar al organismo de los seres humanos, ser transportadas hasta el cerebro y originar enfermedades como Parkinson y Alzheimer (Calderón, Reynoso, Pérez, Mukherjee y Gónzalez, 2017).

Nueva Delhi es una de las ciudades del mundo con una mayor problemática originada en el material particulado; por ello el gobierno centró su atención en las emisiones de vehículos, estableciendo que incluso los pasajeros de dichos automotores se ven afectados por las Partículas Ultrafinas -UFP (Joshua, Apte y Kirchstetter, 2011).

Los datos obtenidos fueron sometidos a un análisis estadístico por medio del método factorial; los resultados presentados se registraron en la Tabla 2. Teniendo en cuenta la representatividad de las muestras, las variables fueron reducidas a PST, $\mathrm{PM}_{10}$ y $\mathrm{PM}_{2.5}$.

\section{Monóxido de Carbono}

Según el estudio de O’Donoghue, Broderick y Delaney (2007), el monóxido de carbono (CO) es el resultado de una combustión incompleta que caracteriza la contaminación móvil, por lo que en Irlanda se utiliza como un marcador para determinar la contribución del tráfico a la contaminación del aire. De ello se desprende que el 4,7\% de los casos de morbilidad hacen referencia a este contaminante en las zonas urbanas.
Huang et al. (2011), realizan un estudio en Beijing (China), donde establecen de acuerdo a la exposición a los contaminantes, la mayor afectación por monóxido de carbono, teniendo en cuenta los casos de pasajeros de bus, taxi y ciclistas, siendo este último el de mayor incidencia en la salud.

\section{Carbono Negro}

El Carbono Negro es un subproducto de la reacción de combustión incompleta que tiene lugar en los vehículos; en Nueva Delhi se ha relacionado esta problemática con la composición del diesel que produce en los seres humanos enfermedades cancerígenas. Teniendo en cuenta lo anterior, la preocupación es aún mayor, ya que el uso del combustible diesel está en aumento y su composición no se encuentra regulada (Apte et al., 2011).

\section{Carbono Elemental}

En Bélgica el uso de combustible diesel va en aumento; en este sentido se han realizado algunas investigaciones relacionadas con el material particulado, sin tener en cuenta que Carbono Elemental es un indicador sensible para determinar las emisiones en este tipo de motores diesel. Por ello se llevó a cabo un estudio que utilizó el modelo de emisión MIMOSA4, a través del cual se plantearon varios escenarios de contaminación, concluyendo que la calidad del combustible incide en un $15 \%$ de las enfermedades respiratorias registradas (Dhondt et al., 2013).

\section{Dióxidos de Azufre y Nitrógeno}

Los dióxidos de azufre y nitrógeno se obtienen en la atmósfera como resultado de la quema de combustibles fósiles, por lo que Mohammad et al. (2016) estudiaron su impacto en la salud de la población de la ciudad de Mashhad utilizado para ello el software AirQ; el resultado evidenció que aproximadamente un 2,6\% de las enfermedades respiratorias son causadas por estas moléculas. 


\section{Consolidado}

De acuerdo con los contaminantes identificados, se evidencia que la proporción de datos es variable tal como se muestra en la Tabla 2; por ello, luego de realizar un análisis estadístico multivariante de la información, resultó que no era representativo incluir los datos para $\mathrm{CO}, \mathrm{NO}_{2}, \mathrm{NO}_{\mathrm{X}}, \mathrm{NO}, \mathrm{CN}, \mathrm{CE}$, $\mathrm{SO}_{2}$.

De acuerdo con lo anterior es evidente que uno de los contaminantes más estudiados a nivel mundial es el material particulado; así mismo, puede esperarse avances en estudios sobre Partículas Ultrafinas (UFP), Carbón Elemental (CE) y Carbono Negro (CN) y su impacto en la salud, ya que las investigaciones en este campo aún se están llevando a cabo.
Tabla 2. Datos primarios obtenidos de las variables evaluadas.

\begin{tabular}{|c|c|}
\hline Contaminante & Datos (\%) \\
\hline PST & 25,25 \\
\hline $\mathrm{PM}_{10}\left(\mu \mathrm{g} / \mathrm{cm}^{3}\right)$ & 30,85 \\
\hline $\mathrm{PM}_{2,5}\left(\mu \mathrm{g} / \mathrm{cm}^{3}\right)$ & 29,27 \\
\hline UFP (partículas $/ \mathrm{cm}^{3}$ ) & 1,43 \\
\hline $\mathrm{CO}(\mathrm{ppm})$ & 3,59 \\
\hline $\mathrm{NO}_{2}(\mathrm{ppb})$ & 2,44 \\
\hline $\mathrm{NO}_{\mathrm{X}}(\mathrm{ppb})$ & 2,15 \\
\hline $\mathrm{NO}(\mathrm{ppb})$ & 2,15 \\
\hline $\mathrm{CN}\left(\mathrm{ug} / \mathrm{m}^{3}\right)$ & 0,72 \\
\hline $\mathrm{CE}\left(\mathrm{ug} / \mathrm{m}^{3}\right)$ & 0,57 \\
\hline $\mathrm{SO}_{2}(\mathrm{ppb})$ & 1,58 \\
\hline
\end{tabular}

Fuente: elaboración propia.

Tabla 3. Datos primarios obtenidos. Asociación entre PM 10, enfermedades y población.

\begin{tabular}{|c|c|c|c|}
\hline Lugar de monitoreo & $\begin{array}{c}\text { PM }_{10}-24 \text { horas } \\
\left(\mu \mathrm{g} / \mathrm{m}^{3}\right)\end{array}$ & $\begin{array}{c}\% \\
\text { Enfermedades }\end{array}$ & Población \\
\hline Monasterevin (Irlanda) (O’Donoghue, Broderick y Delaney, 2007). & 22,68 & $22 \%$ & 4.246 \\
\hline Ciudad Heredia (Costa Rica) (Yura, Kear y Niemeier, 2007). & 25 & $19 \%$ & 138.562 \\
\hline Brisbane (Australia) (Chen, Mengersen y Tong, 2007). & 29,82 & $14 \%$ & 2.177 .000 \\
\hline Quito (Ecuador) (Vlachokostas et al., 2013) & 45,11 & $22 \%$ & 1.619 .000 \\
\hline Madrid (España) (Leganes, 2017). & 79 & $25 \%$ & 3.166 .000 \\
\hline Taichung (Taiwán) (Vlachokostas et al., 2013). & 82,6 & $45 \%$ & 2.797 .000 \\
\hline Mashhad (Irán) (Mohammad et al., 2016) & 82,9 & $43 \%$ & 3.001 .000 \\
\hline Singapur (República de Singapur) (Gupta y Cheong, 2007) & 88,8 & $29 \%$ & 5.612 .000 \\
\hline Yokohama (Japón) (Mohammad et al., 2016) & 91,2 & $51 \%$ & 3.725 .000 \\
\hline Guadalajara (México) (Montaño et al., 2016) & 120 & $40 \%$ & 1495000 \\
\hline Bogotá (Colombia) (Becerra et al., 2014) & 126,4 & $26 \%$ & 8.081 .000 \\
\hline Beijing (China) (Jing et al, 2012) & 147,3 & $86 \%$ & 21.710 .000 \\
\hline Río de Janeiro, Brasil (Tsuruta et al., 2016). & 150 & $28 \%$ & 6.476 .631 \\
\hline El Cairo (Egipto) (Khoder y Hassan, 2008) & 190 & $69 \%$ & 9.700 .000 \\
\hline Hangzhou (China) (Le et al., 2012) & 204 & $82 \%$ & 9.468 .000 \\
\hline Buenos Aires (Argentina) (Martin et al., 2007) & 216 & $54 \%$ & 2.890 .000 \\
\hline Nueva Delhi (India) (Jurado y Mercado, 2017) & 256 & $79 \%$ & 21.750 .000 \\
\hline Santiago de Chile (Prieto et al., 2007) & 150 & $27 \%$ & 5.614 .000 \\
\hline
\end{tabular}

Fuente: elaboración propia. 
En el presente estudio, de las variables que describen el material particulado tiene una mayor importancia la determinación de $\mathrm{PM}_{10}$, por su influencia en la salud de las personas, por eso en la Tabla 3 se relacionan las proporciones de $\mathrm{PM}_{10}$ asociadas con las actividades de transporte en diferentes regiones del mundo, así como su influencia en la salud y su densidad poblacional.

Los datos presentados en la tabla anterior muestran un modelo de regresión lineal vinculado con la densidad de la población y la salud pública en relación con las emisiones de $\mathrm{PM}_{10}$, tal como se presenta en las Figuras 1 y 2.

La influencia de los contaminantes generados por el transporte terrestre convencional, específicamente asociados al material particulado, relacionados con los casos de enfermedades respiratorias y la densidad poblacional se presenta en las Figuras 1 y 2 . Es pertinente mencionar que la densidad poblacional es una variable importante ya que es una medida que permite determinar la influencia de la densidad de tráfico (Eeftens et al., 2015).

De acuerdo con lo anterior la relación entre densidad de población vs $\mathrm{PM}_{10}$ (Figura 1) y $\mathrm{PM}_{10}$ vs Enfermedades Respiratorias (Figura 2), demuestra un comportamiento lineal, ya que el coeficiente de correlación $\mathrm{R}^{2}$ es de 0,565 y 0,583 respectivamente, lo que indica que existe una asociación moderada.

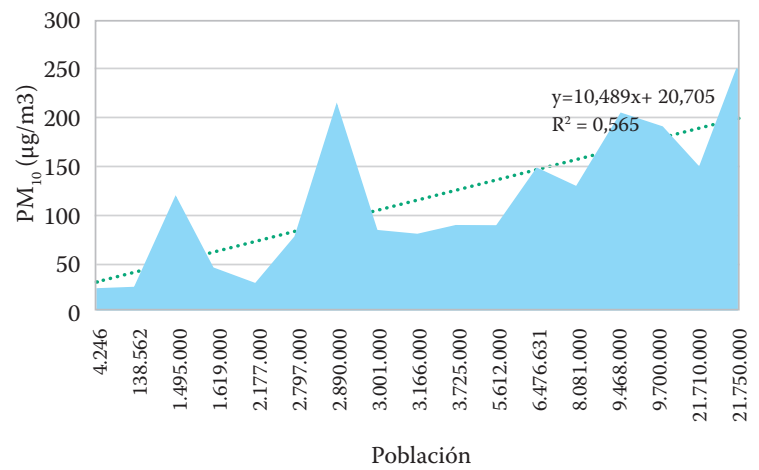

Figura 1. Población vs $\mathrm{PM}_{10}$. Fuente: elaboración propia.

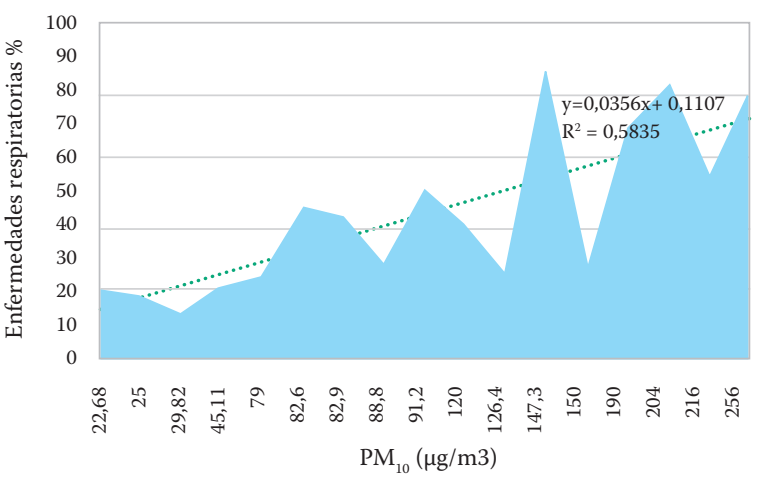

Figura 2. $\mathrm{PM}_{10}$ vs. enfermedades respiratorias. Fuente: elaboración propia.

En la Figura 3 se establece la relación entre las variables que, tal como se identificó en la revisión bibliográfica, están relacionadas con los procesos de combustión del transporte terrestre; en este sentido se establece un comportamiento lineal entre la Población y las Enfermedades Respiratorias, ya que el $R^{2}$ es de 0,5838 .

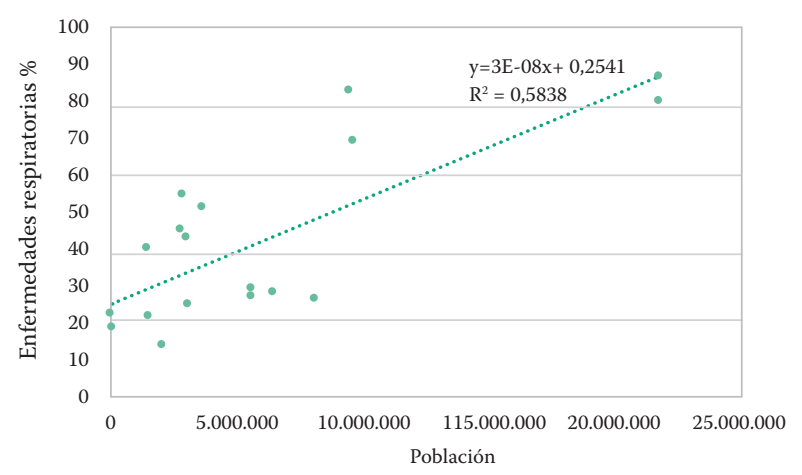

Figura 3. Población vs. enfermedades respiratorias. Fuente: elaboración propia.

De acuerdo con las concentraciones encontradas de $\mathrm{PM}_{10}$ en la revisión bibliográfica, se evidencia que la mayor influencia en la salud pública, en cuanto a las enfermedades respiratorias, se presenta en las regiones evaluadas en China; lo que es acorde con la huella ecológica en dicha región que corresponde a la mayor a nivel mundial (Baddi, 2008).

Desde el punto de vista de la fuente de generación de los contaminantes estudiados (combustión de vehículos), según la investigación de Hak, 
Hallquist, Ljungstro, Svane y Pettersson (2009) en Europa los niveles de la mayoría de los tipos de contaminación han ido disminuyendo; sin embargo, el aporte de material particulado se ha mantenido estable, lo que ha generado efectos sobre la salud como enfermedades respiratorias y cardiovasculares.

Teniendo en cuenta el comportamiento del $\mathrm{PM}_{10}$ en el aire, en el caso de Beijing (China), Huang, Zhuang y Lin (2013) destacan la influencia de la exposición a este material, el cual aumentó directamente con el crecimiento en el parque automotor que se triplicó del 2003 a 2010, generando una fuente importante de contaminación del aire en dicha ciudad. No obstante, el problema es aún mayor por la congestión del tráfico que aumenta el tiempo de exposición a los contaminantes mencionados.

En términos generales se evidencia que las emisiones de $\mathrm{PM}_{10}$ por parte de los vehículos en las diferentes regiones analizadas, presentan un comportamiento lineal con respecto a los casos de enfermedades respiratorias; de la misma forma, la densidad poblacional que se asocia directamente con la densidad de tráfico, presenta una tendencia lineal con respecto a las concentraciones del material particulado.

\section{Contaminantes y límites normativos}

Teniendo en cuenta relación directa que existe entre las concentraciones de $\mathrm{PM}_{10}$ y su influencia en la salud pública, es necesaria la regulación de este tipo de partículas en el aire de las principales ciudades del mundo con el fin de mitigar las consecuencias de su inhalación. Por ello se debe contar con referencias cuantitativas que permitan determinar los niveles de contaminación en exteriores a fin de concretarlos en disposiciones que tengan fuerza legal. La determinación de esos niveles de contaminación debe ser coherente con el riesgo aceptable para la protección de la salud y el bienestar público (OMS, 2000).

Tabla 4. Concentración de emisiones generadas por el transporte terrestre convencional.

\begin{tabular}{|c|c|c|c|c|c|c|c|c|}
\hline \multirow{2}{*}{ Lugar de monitoreo } & \multirow{2}{*}{$\begin{array}{c}\mathrm{PM}_{10}-24 \\
\text { horas } \\
\left(\mu \mathrm{g} / \mathrm{m}^{3}\right)\end{array}$} & \multicolumn{7}{|c|}{ Límites Normativos } \\
\hline & & España & Japón & Colombia & Argentina & México & China & OMS 2005 \\
\hline $\begin{array}{l}\text { Monasterevin (Irlanda) (O'Donoghue, Broderick y } \\
\text { Delaney, 2007). }\end{array}$ & 22,68 & \multirow{18}{*}{50} & \multirow{18}{*}{35} & \multirow{18}{*}{75} & \multirow{18}{*}{150} & \multirow{18}{*}{50} & \multirow{18}{*}{150} & \multirow{18}{*}{50} \\
\hline Ciudad Heredia (Costa Rica) (Yura, Kear y Niemeier, 2007) & 25 & & & & & & & \\
\hline Brisbane (Australia), (Chen, Mengersen y Tong, 2007) & 29,82 & & & & & & & \\
\hline Quito (Ecuador) (Vlachokostas et al., 2013) & 45,11 & & & & & & & \\
\hline Madrid (España) (Leganes, 2017) & 79 & & & & & & & \\
\hline Taichung (Taiwán) (Vlachokostas et al., 2013) & 82,6 & & & & & & & \\
\hline Mashhad (Irán) (Mohammad et al., 2016) & 82,9 & & & & & & & \\
\hline Singapur (República de Singapur) (Gupta y Cheong, 2007) & 88,8 & & & & & & & \\
\hline Yokohama (Japón) (Mohammad et al., 2016) & 91,2 & & & & & & & \\
\hline Guadalajara (México) (Montaño et al., 2016) & 120 & & & & & & & \\
\hline Bogotá (Colombia) (Becerra et al, 2014) & 126,4 & & & & & & & \\
\hline Beijing (China) (Jing et al, 2012) & 147,3 & & & & & & & \\
\hline Río de Janeiro, Brasil (Tsuruta et al, 2016) & 150 & & & & & & & \\
\hline Cairo (Egipto), (Khoder y Hassan, 2008) & 190 & & & & & & & \\
\hline Hangzhou (China) (Le et al., 2012) & 204 & & & & & & & \\
\hline Buenos Aires (Argentina) (Martin et al, 2007) & 216 & & & & & & & \\
\hline Nueva Delhi (India) (Joshua et al, 2011) & 256 & & & & & & & \\
\hline Santiago de Chile (Prieto et al., 2007). & 150 & & & & & & & \\
\hline
\end{tabular}

Fuente: elaboración propia. 
En la Tabla 4 se presenta las concentraciones promedio obtenidas a partir de los estudios encontrados durante la revisión bibliográfica a nivel mundial, relacionados con los contaminantes emitidos por el transporte terrestre convencional; adicionalmente, se encuentra la comparación con los límites normativos de establecidos en España, Japón, Colombia y Argentina.

De acuerdo con lo anterior, los límites normativos refieren un comportamiento directamente proporcional con respecto a la contaminación por $\mathrm{PM}_{10}$ en la región respectiva, es decir, a un mayor nivel de contaminación mayor es el límite normativo aplicado en cada región; no obstante este comportamiento puede estar asociado a las condiciones naturales en cada zona como la topografía, velocidad y dirección de los vientos. También se evidencia, al contrario, que los mayores límites normativos propician la mayor generación de este tipo de contaminantes (Hak et al, 2009).

Las normas de calidad del aire generalmente pasan por un proceso de revisión; en el caso de Santiago de Chile el comité estuvo conformado por el representante de Ministerios y Servicios públicos del Sistema Nacional de Gestión Ambiental y el Comité Ampliado por representantes del mundo académico. El procedimiento de dictación de normas inicia con la elaboración del anteproyecto, seguido del proceso de consulta y el proceso de elaboración del proyecto de norma definitivo, para finalmente ser sometido a consideración del Presidente de la República (Matus y Lucero, 2002).

En otros casos, como el España y México, la normatividad o los estándares relacionados con la calidad del aire en lo que respecta al material particulado, se asumen teniendo en cuenta los límites regulados por la OMS (2005); no obstante, es conveniente que los límites normativos también tengan en cuenta el comportamiento de los contaminantes y la dispersión de los mismos en cada región del mundo.

En Bogotá (Colombia), teniendo en cuenta la influencia de la calidad del aire en la salud humana, en el año 2017 se actualizó la normatividad, implementando límites más estrictos sobre la concentración de partículas contaminantes, decretando niveles que requieren de prevención, alerta o emergencia (Villalobos, Sánchez y Arriero, 2016).

Se puede mencionar que en la mayoría de los casos estudiados en esta revisión, la aplicación de la normativa es posible a través del sistema de vigilancia de los contaminantes del aire que, en concentraciones establecidas, permite generar diferentes alertas sobre su impacto en la salud pública.

\section{Técnicas y métodos utilizados en la medición de contaminantes}

Para la toma de muestras se tuvo en cuenta, previamente, que las mediciones se realizaran en la mayoría de los casos en avenidas principales, determinando el punto estratégico que representara con una mayor claridad, la influencia de los vehículos de transporte terrestre en la calidad del aire; por lo que, como en el caso de Bogotá (Villalobos, Sánchez y Rodríguez, 2016), el material particulado $\left(\mathrm{PM}_{10}\right.$ y $\left.\mathrm{PM}_{2,5}\right)$ presenta un comportamiento directamente proporcional con el número de vehículos transitado, dicho elemento se estableció como punto de monitoreo. En otros casos, de acuerdo con Huang et al. (2011), se implementó un equipo de medida portátil realizando mediciones durante una ruta determinada.

En el caso de Río de Janeiro (Brasil), las mediciones se realizaron a través de la instalación de cuatro estaciones de monitoreo automático que son operadas por la Agencia Estatal (INEA); los datos obtenidos fueron cotejados con el índice de calidad calculado y solamente se reportaron los contaminantes que representan las peores condiciones (Tsuruta, Carvalho, Silva y Arbilla, 2016).

De otro lado, el caso de estudio de Hangzhou (China) refiere la implementación de un contador de partículas de condensación (CPC) para medir 
la cantidad del material particulado; en esta investigación sobresale la aplicación del modelo ARIMA, para evaluar el comportamiento de los contaminantes emitidos por el transporte terrestre convencional en la atmósfera (Le et al., 2012).

Con el fin de recolectar la información acerca de las técnicas y métodos implementados en los casos de estudio mencionados en este artículo, en la Tabla 4 se presenta la proporción de estudios que aplican las diferentes metodologías ya expuestas.

Tabla 4. Metodología de muestreo.

\begin{tabular}{|l|c|}
\hline \multicolumn{1}{|c|}{ Técnicas y métodos } & $\begin{array}{c}\text { Estudios } \\
\text { aplicados (\%) }\end{array}$ \\
\hline Red de monitoreo de calidad del aire & 16 \\
\hline Equipos portátiles & 37 \\
\hline Modelación de la calidad del aire & 21 \\
\hline Análisis gravimétricos & 26 \\
\hline
\end{tabular}

Fuente: elaboración propia.

\section{Conclusiones}

A partir de la revisión bibliográfica en torno a la calidad del aire y la influencia de las emisiones generadas por el transporte terrestre, se evidencia que los contaminantes más estudiados por su relación con la salud humana es el material particulado (PST, $\mathrm{PM}_{10}$ y $\mathrm{PM}_{2,5}$ ); en este sentido, por medio de las concentraciones identificadas a nivel mundial, se evidencia una función lineal entre la densidad poblacional y el $\mathrm{PM}_{10}$, así como entre el $\mathrm{PM}_{10}$ y la tasa de enfermedades respiratorias; demostrando una incidencia en casos de enfermedades respiratorias superior al $80 \%$ en ciudades de China e India.

Con respecto a los demás contaminantes generados por los procesos de combustión en las actividades de transporte, es notoria una tendencia creciente y relativamente reciente en relación con las emisiones, por parte del transporte terrestre, de Partículas Ultrafinas (UFP), Carbono Elemental y Carbono Negro.
Desde el punto de vista normativo, los límites más flexibles se presentan en Argentina y China, con un valor de $\mathrm{PM}_{10}$ diario de $150 \mu \mathrm{g} / \mathrm{m}^{3}$; tomando este valor como referencia, se identifica el incumplimiento en Río de Janeiro (Brasil), El Cairo (Egipto), Hangzhou (China), Buenos Aires (Argentina), Nueva Delhi (India) y Santiago de Chile (Chile). Al contrario, el límite más restrictivo se presenta en Japón, el cual es de $35 \mu \mathrm{g} / \mathrm{m}^{3}$; lo anterior indica que en más del $80 \%$ de los casos de estudio analizados, se incumpliría con este requerimiento normativo.

Con respecto a las técnicas y métodos empleados para la medición de partículas contaminantes, en los casos de estudio analizados sobresale la utilización de equipos portátiles en un 37\% y se evidencia que a lo largo del tiempo disminuye la utilización de análisis gravimétricos; por ello, en la actualidad, se aumenta la utilización de modelación de la calidad del aire, método que alcanza un $21 \%$.

\section{Referencias bibliográficas}

Arango, J. (2009). Calidad de los combustibles en Colombia. Revista de Ingeniería, 29, pp. 100-108. Recuperado de: [http://www.scielo. org.co/pdf/ring/n29/n29a13.pdf].

Arciniégas, A. (2012). Diagnóstico y control de material particulado: partículas suspendidas totales y fracción respirable $\mathrm{PM}_{10}$. Luna Azul, 34, pp. 195-213. Recuperado de: [http://www. scielo.org.co/pdf/luaz/n34/n34a12.pdf].

Baddi, M. (2008). La huella ecológica y sustentabilidad. Dacna: International Journal of Good Conscience, 3, pp. 672-678. Recuperado de: [http://www.spentamexico.org/v3-n1/3(1)\%20 672-678.pdf].

Becerra, L., Miranda, V., Hernández, L., Barraza, A., Junger, W., Hurtado, M. y Romieu, I. (2014). Effect of particulate matter less than $10 \mu \mathrm{m}$ (PM10) on mortality in Bogotá, Colombia: a time.series analysis, 1998-2006. Salud Pública de México. 56(4), pp. 363-370. Recuperado de: [scielo.org.mx/pdf/spm/v56n4/v56n4a10.pdf]. 
Calderón, L., Reynoso, R., Pérez, B., Mukherjee, P. y Gónzalez, A. (2017). Combustion derived nanoparticles, the neuroenteric system, cervical vagus, hyperphosphorylated Alpha synuclein and tau in Young Mexico City residents. Environmental Research, 159, pp. 186-201. DOI: [http://dx.doi.org/10.1016/j. envres.2017.08.008].

Cengel, Y. (2006). Termodinámica. México: McGraw Hill.

Chen, L., Mengersen, K. y Tong, S. (2007). Spatiotemporal relationship between particle air pollution and respiratory emergency hospital admissions in Brisbane, Australia. Science of the total environment. 373(1), pp. 57-67. DOI: [https://doi.org/10.1016/j. scitotenv.2006.10.050].

Cursach, J., Rau, J., Tobar, C. y Ojeda, J. (2012). Estado actual del desarrollo de la ecología urbana en grandes ciudades del sur de Chile. Revista de Geografía Norte Grande, 52, pp. 57-70. DOI: [http://dx.doi.org/10.4067/ S0718-34022012000200004].

Dhondt, S., Beckx, C., Degraeuwe, B., Lefebvre, W., Kochan, B., Bellemans, T., Panis, L., Macharis, C. y Putman, K. (2012). Integration population mobility in the evaluation of air quality measures on local and regional scales. Atmospheric Environment. 59, pp. 67-74. DOI: [https://doi. org/10.1016/j.atmosenv.2012.04.055].

Eeftens, M., Phuleria, H., Meier, R., Aguilera, I., Corradi, E., Davey, M., Ducret, R., Fierz, M., Gehrig, R., Ineichen, A., Keidel, D., Probst, N., Ragettli, M., Shindler, C., Kunzli, D. y Tsai, M. (2015). Spatial and temporal variability of ultrafine particles, NO2, PM2.5, PM2.5 absorbance, PM10 and PMcoarse in Swiss study areas. Atmospheric Environment. 111, pp. 60-70. DOI: [http://dx.doi.org/10.1016/j. atmosenv.2015.03.031].

Gaviria, C., Benavides, P. y Tangarife, C. (2011). Contaminación por material particulado $\left(\mathrm{PM}_{2,5}\right.$ y $\mathrm{PM}_{10}$ ) y consultas por enfermedades respiratorias en Medellín (2008-2009). Revista Facultad
Nacional de Salud Pública, 29, pp.241-250. Recuperado de: [http://www.scielo.org.co/pdf/ rfnsp/v29n3/v29n3a04.pdf].

Gómez, O. y Vigano, R. (2007). Propuesta de solución bimodal al problema de la contaminación vehicular urbana. Revista de ingeniería $e$ investigación. 27 (3), pp. 143-148. Recuperado de: [http://www.scielo.org.co/pdf/iei/v27n3/ v27 n3a16.pdf].

Gupta, A. y Cheong, D. (2007). Physical characterization of particulate matter and ambient meteorological parameters at different indoor - outdoor locations in Singapore. Building and Environment, 42 (1), pp. 237-245. DOI: [https:// doi.org/10.1016/j.buildenv.2006.02.017].

Hak, C., Hallquist, M., Ljungstro"m, E., Svane, M. y Pettersson, J. (2009). A new approach to in-situ determination of roadside particle emission factors of individual vehicles under conventional driving conditions. Atmospheric Environment, 43, pp. 2481-2488. DOI: [https:// doi.org/10.1016/j.atmosenv.2009.01.041].

Hao, Y., Meng, X., Yu, X., Lei, M., Li, W., Shi, F., Yang, W., Zhang, S. y Xie, S. (2018). Characteristics of trace elements in $\mathrm{PM}_{2.5}$ and $\mathrm{PM}_{10}$ of Chifeng, northeast China: Insights into spatiotemporal variations and sources. Atmospheric Research, 213, pp. 550-561. DOI: [https://doi. org/10.1016/j.atmosres.2018.07.006].

Huang, K., Zhuang, G. y Lin, Y. (2013). How to improve the air quality over megacities in China: pollution characterization and source analysis in Shanghai before, during, and after the 2010 World Expo. Atmospheric Chemistry and Physics, 13(12), pp. 5927-5942. DOI: [https://doi.org/10.5194/acp-13-5927-2013].

Jing, H., Furong, D., Shaowei, W. y Xinbiao, G. (2012). Comparisons of personal exposure to PM2.5 and CO by different commuting modes in Beijing, China. Science or the total environment. 425, pp. 52-59. DOI: [https://doi. org/10.1016/j.scitotenv.2012.03.007]. 
Joshua, S., Apte, T., Reich, A., Deshpande, S., Kaushik, G., Chel, A., Marshall, J. y Nazaroff, W. (2011). Concentrations of fine, ultrafine, and black carbon particles in auto-rickshaws in New Delhi, India. Atmospheric Environment, 45 (26), pp. 4470-4480. DOI: [https://doi.org/10.1016/j. atmosenv.2011.05.028].

Jurado, M. y Mercado, D. (2017). Revisión sistemática de técnicas no convencionales para la evaluación de la calidad del agua de ríos contaminados con plaguicidas. Entre Ciencia e Ingeniería, 21, pp. 56-65. Recuperado de: [http:// www.scielo.org.co/pdf/ecei/v11n21/1909-8367ecei-11-21-00056.pdf].

Khoder, M. y Hassan, K. (2008). Weekday/weekend differences in ambient aerosol level and chemical characteristics of water-soluble components in the city centre. Atmospheric Environment, 42, pp. 7483-7493. DOI: [https://doi.org/10.1016/j. atmosenv.2008.05.068].

Le, J., Yun, Z., Yi-Ping, Z., Mei-Bian, Z. y Dean, B. (2012). An application of ARIMA model to predict submicron particle concentrations from meteorological factors at a busy roadside in Hangzhou, China. Science of The Total Environment, 425, pp.336-345. Recuperado de: [https:// www.sciencedirect.com/science/article/abs/pii/ S0048969712003853?via\%3Dihub].

Leganes, M. (2017). La calidad del aire en la ciudad de Madrid en 2017. Ecologistas en acción, 93, pp. 1-25. Recuperado de: [https://spip. ecologistasenaccion.org/IMG/pdf/info-calidadaire-madrid-2017.pdf].

Llanes, E., Rocha, J., Peralta, D.y Leguísamo, J. (2018). Evaluación de emisiones de gases en un vehículo liviano a gasolina en condiciones de altura. Caso de estudio Quito, Ecuador. Enfoque UTE, 9(2), pp. 149-158. Recuperado de: [http://scielo. senescyt.gob.ec/scielo.php?script=sci_arttext\& pid $=$ S1390-65422018000200149].

Martin, S., Dawidowski, L., Mandalunis, P., Cereceda, F. y Tasat, D. (2007). Characterization and biological effect of Buenos Aires urban air particles on mice lungs. Environmental Research, 105 (3), pp. 340-349. DOI: [https:// doi.org/10.1016/j.envres.2007.04.009].

Martins, N. y Carrilho, G. (2018). Impact of PM2.5 in indoor urban environments: A review. Sustainable Cities and Society, 42, pp. 259-275. DOI: [https://doi.org/10.1016/j.scs.2018.07.011].

Matus, P. y Lucero, R. (2002). Norma Primaria de Calidad del aire. Revista Chilena de enfermedades respiratorias, 18(2), pp. 259-275. DOI: [http://dx.doi.org/10.4067/ S0717-73482002000200006].

Mendoza, M. y Lombardo, M. (2009). El clima urbano de ciudades subtropicales costeras atlánticas: el caso de la conurbación de Florianópolis. Revista de geografía Norte Grande,44, pp. 129-141. DOI: [http://dx.doi.org/10.4067/ S0718-34022009000300007].

Mohammad, M., Derakhshan, Z., Allahabadi, A., Ahmadi, E., Oliveri, G., Ferrante, M. y Aval, H. (2016). Mortality and morbidity due to exprosure to outdoor air pollution in Mashhad metrópolis, Iran. The AirQ model approach. Environmental Research, 151, pp. 451-457. DOI: [https://doi.org/10.1016/j.envres.2016.07.039].

Montaño, F., Davydova, V., Chávez, G., Gallardo, P. y Orozco, M. (2016). $\mathrm{PM}_{10}$, y $\mathrm{O}_{3}$ como factores de riesgo de mortalidad por enfermedades cardiovasculares y neumonía en la zona Metropolitana de Guadalajara, Jalisco, México. Ingeniería Revista Académica. 20(1), pp. 14-23. Recuperado de: [http://www.redalyc.org/articulo.oa?id=46750927002].

O’Donoghue, R., Broderick, B. y Delaney, K. (2007). Assessing the impacts of infrastructural road changes on air quality: A case study. Transportation Research Part D: Transport and Environment, 12(8), pp. 529-536. DOI: [https:// doi.org/10.1016/j.trd.2007.07.009].

OMS (2000). Guías para la calidad del aire. CEPIS. 4.110. Organización Mundial de la Salud, pp. 90-110. Recuperado de: [http://www.ingenieroambiental.com/4014/guiasaire.pdf]. 
OMS (2005). Guías de calidad del aire del OMS relativas al material particulado, el ozono, el dióxido de nitrógeno y el dióxido de azufre. CEPIS. 6.02. Organización Mundial de la Salud, pp. 10-20. Recuperado de: [https://www.who. int/phe/health_topics/AQG_spanish.pdf].

Prieto, M., Mancilla, P., Astudillo, P., Reyes, A. y Roman, O. (2007). Exceso de morbilidad respiratoria en niños y adultos mayores en una comuna de Santiago con alta contaminación atmosférica por partículas. Revista Médica de Chile, 135(2), pp. 221-228. DOI: [http://dx.doi. org/10.4067/S0034-98872007000200012].

Tsuruta, F., Carvalho, J., Silva, C. y Arbilla, G. (2016). Air Quality Indexes in the City of Rio de Janeiro During the 2016 Olympic and Paralympic Games. Journal of the Brazilian Chemical Society., 29(6), pp. 1291-1303. DOI: [http:// dx.doi.org/10.21577/0103-5053.20170226].

Villalobos, O., Sarmiento, D. y Rodríguez, D. (2016). The $\mathrm{PM}_{10}$ pollutant and impact on health in the locality of Tunjuelito. Visión Electrónica, 10(2), pp. 274-283. DOI: [https://doi. org/10.14483/22484728.1174].

Vlachokostas, Ch., Michailidou, A., Spyridi, D. y Moussiopoulos, N. (2013). Building statistical associations to forecast ethylbenzene levels in European urban-traffic environments. Environmental Pollution, 177, pp. 125-134. DOI: [http:// dx.doi.org/10.1016/j.envpol.2013.02.011].

Yura, E., Kear, T. y Niemeier, D. (2007). Using CALINE dispersión to assess vehicular PM2.5 emissions. Atmospheric Enviroment, 41(38), pp. 8747-8757. DOI: [https://doi.org/10.1016/j. atmo senv.2007.07.045].

Zambrano, E. y Rojas, I. (2009). Calidad del aire y su incorporación en la planeación urbana: Mexicali, Baja California, México. Estudios Fronterizos, 10(20), pp. 79-102. Recuperado de: [http:// www.scielo.org.mx/scielo.php?script=sci_artte xt\&pid=S0187-69612009000200003]. 